\title{
Survival thresholds and mortality rates in adaptive dynamics: conciliating deterministic and stochastic simulations
}

\author{
Benoît Perthame $^{\mathrm{a}, \mathrm{b}}$, Mathias Gauduchon ${ }^{\mathrm{c}}$ \\ ${ }^{a}$ UPMC Univ Paris 06, UMR 7598, Laboratoire Jacques-Louis Lions, F-75005, Paris, France et Institut Universitaire de \\ France \\ ${ }^{b}$ CNRS, UMR 7598, Laboratoire Jacques-Louis Lions, F-75005, Paris, France \\ ${ }^{c}$ Laboratoire de Microbiologie, Géochimie et Écologie Marines, UMR 6117, Centre d'Océanologie de Marseille, Université de \\ la Méditerranée - CNRS, Campus de Luminy, Case 901, 13288 MARSEILLE Cedex 09
}

\begin{abstract}
Deterministic population models for adaptive dynamics are derived mathematically from individual centered stochastic models in the limit of large populations. However numerical simulations of both models do not fit and give rather different behaviors in terms of evolution speeds and branching patterns. Stochastic simulations involve extinction phenomenon operating through demographic stochasticity, when the number of individual 'units' is small. We include a similar notion in the deterministic models, a survival threshold, which allows phenotypical traits in the population to vanish when represented by few 'individuals'. Based on numerical simulations, we show that the survival threshold changes drastically the solution ; (i) the evolution speed is much slower, (ii) the branching patterns is reduced continuously; (iii) they are comparable to the stochastic simulations. The rescaled models can also be analyzed theoretically. One can recover the concentration phenomena on well separated Dirac masses through the constrained Hamilton-Jacobi equation in the limit of small mutations and large observation times.
\end{abstract}

Key words: evolution, Adaptative Dynamics, Hamilton-Jacobi equation, asymptotic analysis, demographic stochasticity, individual based model, evolutionary branching

\section{Introduction}

The theory of adaptive dynamics aims at describing biological evolution driven by mutations and selection. It covers several formalisms as the geometrical classification of evolutionary stable states $([28,25,24$, $18,15])$ and the canonical equations of adaptive dynamics $([14,9,29])$. These theories aim at describing the dynamics of the dominant trait in the population, that we will call the 'fittest' trait. Other points of view aim at describing the full population and how this trait is selected. Most popular are stochastic, or individual centered, models $([18,21,10,8])$. In the limit of large population, integro-differential equations or partial differential equations can be derived $([4,20,10])$. These models have also been used and studied intensively $([5,6,13,21,22,27])$ and it is also possible to derive the adaptive evolution of the fittest trait assuming rare or small mutations. This leads to perform an asymptotic analysis that has been proposed in [16] and which has been extended in $[2,3,23,7]$. Its interest relies on the relaxed Hamilton-Jacobi equation that describes the dynamics of the fittest trait, similar to mathematical objects arising in large deviations or front propagation $[17,1,31,19]$.

Direct numerical simulations have also been performed in both the stochastic and deterministic situations described before. Eventhough it is theoretically proved that there is convergence of stochastic models towards the nonlinear deterministic models in various limits for large populations, one can observe that typical stochastic and deterministic simulations do not fit and give rather different behaviors in terms of evolution

URL: benoit.perthame@upmc.fr (Benoît Perthame), mathias.gauduchon@univmed.fr (Mathias Gauduchon) Preprint submitted to Theoretical Population Biology

September 17, 2008 
speeds and branching patterns. It is noticeable that stochastic simulations, using a finite size population, involve extinction phenomenon operating through demographic stochasticity which acts drastically on small populations. In opposition to continuous models, these simulations involve a unit for minimal survival population size, which corresponds to a single individual. The notion of demographic stochasticity does not occur in general in deterministic population models although its importance has been pointed out in several papers, see for instance $[32,11]$ and the references therein.

In this paper we propose to include a similar notion in the deterministic models, a survival threshold, which allows some phenotypical traits of the population to vanish when represented by too few individuals. Our aim is to show the importance of survival thresholds in deterministic models for adaptive evolution. In particular, taking into account for statistical effects on small populations with this notion, we can compare the results of the deterministic population evolution with stochastic simulations. The importance of survival threshold is demonstrated through a simple and standard Lotka-Volterra model example that we borrow from [29]. Yet, we perceive that the results should overpass the specific model we study and have a very broad scope. Several characteristic features become different: (i) the evolution speed is much lower with a survival threshold because many invading mutants cannot survive with the new death rates due to the survival threshold; (ii) further, population tails disappear and the size of represented traits remains finite, avoiding the unrealistic effect that extremely small populations can find a favorable adaptive landscape and suddenly dominate the population; (iii) branching patterns become closer to stochastic simulations, they are smoother and the model avoids jumps of infinitely rapid branching, leading to numerical solutions in accordance with demographic stochasticity effects.

In a different context, the author in [33] proposed corrections to the classical deterministic models for spatially structured populations in order to fit to individual-based stochastic simulations. Nevertheless, beside our special focus on models for evolution and resulting evolutionary patterns, we propose here a novel approach in term of an explicit new form of deterministic models.

The survival threshold introduces a new scale to the problem, the minimal size of the population that may survive, in addition to the mutation rate or mutation average size. We show that in a certain range (a so-called distinguished limit) when both this survival threshold and the mutation rate are small, the asymptotic analysis can still be performed based on Constrained Hamilton-Jacobi equation, that gives rise a new and interesting theoretical object. However a careful analysis of the numerical transitions between different branching patterns leads us to think that other natural scales might exist in the model, related to the size of the survival threshold.

\section{Survival threshold}

Consider a general deterministic model for adaptive evolution. When $n(t, x)$ stands for the distribution at time $t(>0)$ of phenotypic trait of value $x(\in \mathbb{R})$, one can model its evolution through several types of classical PDEs, see for example [6, 27, 5, 29, 16, 22, 21, 13],

$$
\frac{\partial}{\partial t} n(t, x)=r(x,[n(t, .)]) n(t, x)+M(x,[n(t, .)]) .
$$

Here $r(x,[n(t,)]$.$) is the selection term (reaction) and denotes the per capita growth rate at time t$ of individuals of type $x$ in the population distribution $[n(t,)$.$] . The mutation term (diffusion, integral operator),$ $M(x,[n(t,)]$.$) , denotes the outcome between incoming mutations from other types in the population distri-$ bution $[n(t,)$.$] to type x$ and outgoing mutations from type $x$. We consider in the following two general and classical forms of the mutation term. Such modeling has been broadly used so far, but they often show up unsatisfactory when they are compared to stochastic individual based simulations. Results differ not only for evolution speeds or quantitative characteristics, but also for qualitative evolution outcomes. Classical deterministic models for example completely do not handle extinction phenomenon operating through demographic stochasticity, leading to 'tails' of populations with very few individuals. Also they hardly show regular branching patterns corresponding to stochastic models: spots often arise suddenly from the afore mentioned tails as depicted in Figure 1, an effect that is often observed in deterministic simulations, see $[13,23,3]$ for instance. 
In our motivation to respond to this inadequacy, we introduce an additional corrective term, the mortality threshold, changing the usual deterministic formulation to

$$
\frac{\partial}{\partial t} n(t, x)=r(t, x,[n(t, .)]) n(t, x)-\sqrt{\bar{n} n(t, x)}+M(t, x,[n(t, .)])
$$

The square root enables the population to vanish for some traits when the population density is too low, thus mimicking the extinction probability due to demographic stochasticity in small sub-populations. At the extreme it means that we do not want to have densities corresponding to less that one individual.

In order to further study the effect of the mortality threshold, we consider more specifically, following [6, 27, 29, 22], the so-called 'Lotka-Volterra' model for intra-specific competition in a deterministic population, with the selection term of the form $r(x,[n(t,)])=.\left(R(x)-\left(K_{\sigma} * n(t,).\right)(x)\right)$ and the mutation term $M(x,[n(t,:])$ set as $\mu \Delta n(t, x)$. Incorporating the survival threshold, we get:

$$
\frac{\partial}{\partial t} n(t, x)=\left(R(x)-\left(K_{\sigma} * n(t, .)\right)(x)\right) n(t, x)-\sqrt{\bar{n} n(t, x)}+\mu \Delta n(t, x) .
$$

The first term of the selection, $R(x)$, describes the density independent part of the invasion exponent. A typical example, that we borrow from [29] and use in numerical solutions, is given by

$$
R(x)=1-x^{2} .
$$

The localized convolution kernel $K_{\sigma}$ describes the competition between similar individuals in terms of $x$. Typically, we choose it, still following [29], as a gaussian

$$
K_{\sigma}(x)=\frac{1}{\sqrt{2 \pi \sigma}} e^{-|x|^{2} /(2 \sigma)} .
$$

It is also standard to describe the mutations by a Laplace term and $\mu$ denotes the mutation frequency and size. Analysis and derivation of this model, in particular from stochastic individual based systems, can be found in $[29,4,8,10,13]$. The derivation from the individual scale also shows that an alternative way to take into account the mutations is to use an integral kernel. We extend our analysis to such kernels in Section 5 .

Adimensionalization in $n$ of equation (3) (replace $n$ by $\lambda n$ and $\bar{n}$ by $\lambda / \bar{n}$ ), shows that we can avoid to introduce a ratio coefficient between the two terms $R$ and $K_{\sigma} * n$ of the invasion exponent. In order to observe the long term effect of evolution, we assume some smallness in mutations that can be modeled in choosing a small coefficient $\mu$; then we rescale time so that we pass from the generation time in (3) to the evolution time scale. That is our motivation to set $\mu=\varepsilon^{2}$ and replace $t$ by $t / \varepsilon$, considering thereafter small $\varepsilon$ values. With the notation $n_{\varepsilon}(t, x)=n\left(\frac{t}{\varepsilon}, x\right)$, we arrive to the equation

$$
\frac{\partial}{\partial t} n_{\varepsilon}(t, x)=\frac{1}{\varepsilon}\left(R(x)-\left(K_{\sigma} * n_{\varepsilon}(t, .)\right)(x)\right) n_{\varepsilon}(t, x)-\frac{1}{\varepsilon} \sqrt{\bar{n} n_{\varepsilon}(t, x)}+\varepsilon \Delta n_{\varepsilon}(t, x) .
$$

In the numerical solutions, we complete it with an initial data of monomorphic type

$$
n_{\varepsilon}(t=0)=\frac{\alpha}{\sqrt{2 \pi \varepsilon}} e^{-\left(x-x_{0}\right)^{2} /(2 \varepsilon)} \underset{\varepsilon \rightarrow 0}{\longrightarrow} \alpha \delta\left(x-x_{0}\right) .
$$

We point out that accelerating time also changes the nonlinear effect of stochastic mortality and thus we can expect that the mortality threshold $\bar{n}$ should also be rescaled in this model as we discuss it later. We will then refer to $\bar{n}_{\varepsilon}$.

\section{Evolution speed, branching patterns and compatibility with stochastic simulations}

Several theoretical works derive deterministic population models in the limit of large populations, with various assumptions on the mortality, mutations and birth rates, see for instance [10]. However, stochastic 
descriptions introduce naturally the individual reference, a notion that disappears in classical deterministic models because changing $n$ in $\lambda n$ leaves them invariant. The new parameter $\bar{n}$, in the deterministic model, restores an order of magnitude corresponding to a real individual. With this in mind we present numerical simulations where $\bar{n}$ varies, in face of stochastic simulations involving different population sizes. In first approximation, the instantaneous total population $\int n(t, x) d x$ is roughly determined by the selection term $r(x,[n(t)])$ mainly, therefore diminishing (increasing) $\bar{n}$ hardly affects the value of $\int n(t, x) d x$ but increases (diminishes) the number of individuals this value represents.

We begin with a short presentation of the numerical algorithms we used, both deterministic and stochastic. Then, we analyse the effect of the survival threshold in the deterministic population model and compare with stochastic simulations.

\subsection{Numerical algorithms}

Being given a uniform grid $\left(x_{i}\right)_{i \in \mathbb{Z}}$ with size $h$, a standard semi-discrete version of the population model (6) reads as

$$
\left.\frac{d}{d t} n_{i}(t)-\frac{\varepsilon}{h^{2}}\left[n_{i+1}(t)+n_{i-1}(t)-2 n_{i}(t)\right]=\frac{n_{i}(t)}{\varepsilon}\left(R_{i}-h \sum_{j} K_{i j} n_{j}(t)\right)\right)-\frac{1}{\varepsilon} \sqrt{\bar{n} n_{i}(t)}
$$

with $R_{i}=R\left(x_{i}\right), K_{i j}=K_{\sigma}\left(x_{i}-x_{j}\right)$. This is the basis of our deterministic population model. It is completed with semi-implicit time discretization and we have tested several variants which leads to the same numerical solutions. The simplest consists in choosing a time step $\Delta t$ and taking

$$
\begin{aligned}
n_{i}^{k+1}-\frac{\varepsilon \Delta t}{h^{2}}\left[n_{i+1}^{k}+n_{i-1}^{k}-2 n_{i}^{k}\right]=n_{i}^{k}+ & \Delta t \frac{n_{i}^{k}}{\varepsilon}\left(R_{i}-h \sum_{j} K_{i j} n_{j}^{k}-\sqrt{\frac{\bar{n}}{\alpha+n_{i}^{k}}}\right)_{+} \\
& +\Delta t \frac{n_{i}^{k+1}}{\varepsilon}\left(R_{i}-h \sum_{j} K_{i j} n_{j}^{k}-\sqrt{\frac{\bar{n}}{\alpha+n_{i}^{k}}}\right)_{-} .
\end{aligned}
$$

The values of $\varepsilon$ under consideration make that the Laplace term can be treated explicitly. The birth-death term is the real numerical difficulty and this scheme is unconditionally stable. Its simplicity, in particular in terms of its effective implementation is an advantage that is counterbalanced by the necessity to introduce a small numerical parameter $\alpha$ to avoid division by zero. It has to be checked a posteriori that this parameter does not influence the numerical solution. As mentioned earlier several variants are possible and one of them, leading to solve a second order equation consists in choosing the square root term implicitly.

Regarding the stochastic framework, our simulations use ZEN, an evolution dedicated software, which can be found in [26] with all the associated modeling formalism. Each individual $i$ has (density-dependent) birth and death instantaneous propabilities. The birth rate has been chosen as the positive term of the discrete form equivalent of the reaction term $r$ in (1), with its precise form from (4), that is

$$
b_{i} \propto R\left(x_{i}\right)=1-x_{i}^{2},
$$

with $x_{i}$ the trait of individual $i$. Similarly, the death rate is derived from the negative part of $r$, that is, from $(5)$,

$$
d_{i} \propto \frac{1}{K} \sum_{j} K_{\sigma}\left(x_{j}-x_{i}\right)=\frac{1}{K} \sum_{j} \frac{1}{\sqrt{2 \pi \sigma}} e^{-\left|x_{j}-x_{i}\right|^{2} /(2 \sigma)} .
$$

The new term $K$ stands for the "carrying capacity" and roughly determines the total number of individuals in the population, what let us choose different population sizes. Evolution is modeled through a constant probability $m$ that each birth from an individual $i$ is accompanied by a mutation of the trait $x_{i}$ in which case the trait of the offspring differs from its parent trait $x_{i}$ by a value that follows a normal distribution of standard deviation $\sigma_{m}$. 


\subsection{Numerical simulations}

First of all, we present quantitative aspects derived from direct numerical simulations of the solution to deterministic equation (6). In these rescaled variables, we find evolutionary times of order 1 , in accordance with the proposed time scale. And, as expected in adaptive dynamics, we obtain very concentrated solutions (on the scale $x \approx \varepsilon$ ) with possible branching patterns. We indicate how these rough features depend on the survival threshold $\bar{n}$.

A major effect can be observed on numerical solutions of (6) in Figure 2; the survival threshold determines the branching pattern. Choosing $\bar{n}$ too large leads to extinction (not shown on figure). For sufficiently large $\bar{n}$, we obtain a monomorphic pattern and then, as $\bar{n}$ diminishes, one can observe symmetric branchings (compared to those depicted in Figure 3) to a dimorphic and even trimorphic population. When the survival threshold $\bar{n}$ is too small we recover that spontaneous jumps replace the continuous branching as mentioned earlier (see Figure 1).

This effect is completely in adequacy with the branching paterns we observe in stochastic simulations performed using the same modeling assumptions on selection rate and competition term. We give in Figure 4 two stochastic simulations corresponding to two cases presented in Figure 2. On this time scale, we can observe that the branching patterns are now in remarkable agreement. We recover in both simulations that the first branching point only occurs if the survival threshold becomes small enough. When the classical model only predicts the evolution toward trimorphism, the stochastic simulations exhibit in addition reduced branching patterns - intermediate persistent dimorphism and even asymptotic monomorphism - as the solutions from the mortality threshold model do. In accordance to the predictions, the patterns obtained for small $\bar{n}$ are similar (in terms of number of branches) to the patterns obtained with stochastic simulations for high population sizes. An other common feature that emerges relies in the smooth branching process we recover in the new approach, that corresponds to what we observe in the stochastic simulations.

A last striking effect of the survival threshold is the evolution speed which becomes faster as $\bar{n}$ diminishes. On Figure 2, one can observe that branching occurs faster, because the 'fittest trait' reaches the branching value $x=.5$ faster (notice that the four numerical solutions are shown on different final times). This is again in adequacy with stochastic simulations in which the population size increases.

These conclusions are confirmed by another set of numerical solutions with a quadratic competition kernel $K_{\sigma}(x)=\kappa_{\sigma}\left(1-\frac{x^{2}}{\sigma^{2}}\right)_{+}$(a probability kernel). Figure 3 shows the branching patterns for $\sigma=1 / 10$. Surprisingly the branching symmetry is lost.

The behavior of our stochastic simulations are in accordance with [11] where the relationship between population sizes and evolutionary branchings has been further explored in a lake ecosystem model. They find that the first branching is first delayed when the carrying capacity of the lake - and thus the population size - gets smaller and is eventually prevented for even smaller populations, what results, as in our case, in asymptotically monomorphic populations.

\section{Asymptotic analysis}

In this section we present a formal analysis of the limiting behavior, as $\varepsilon$ vanishes, of solutions to (6). We extend the asymptotic analysis describing the convergence of solutions to Dirac masses in such a system, following earlier ideas developed in $[16,2,3,7,23,30]$ for the case $\bar{n}=0$ (no survival threshold). In the present context, the questions are to understand the scale for the survival threshold $\bar{n}_{\varepsilon}$ compatible with the analysis, and to analyze the effect on the limit $\varepsilon=0$.

We still consider the scaled version (6) of the deterministic model and we recall that the notion of polymophic populations can be understood as the concentration on the population density as Dirac masses in the limit $\varepsilon \rightarrow 0$

$$
n_{\varepsilon}(t, x) \underset{\varepsilon \rightarrow 0}{\longrightarrow} n(t, x)=\sum_{i} \alpha_{i}(t) \delta\left(x-x_{i}(t)\right) .
$$

This (weak) limit can be described more accurately through the phase function $\varphi_{\varepsilon}$ defined by

$$
n_{\varepsilon}(t, x)=e^{\varphi_{\varepsilon}(t, x) / \varepsilon}
$$


just as the (weak) convergence of the gaussian $\frac{1}{\sqrt{2 \pi \varepsilon}} e^{-|x|^{2} /(2 \varepsilon)}$ to the Dirac mass $\delta(x)$ is better described by the fact that the polynomial $|x|^{2}$ vanishes at a single point.

The choice of the survival threshold is crucial here. Taking $\bar{n}$ as a positive constant is too strong and leads the system to extinction as $\varepsilon$ becomes small. Hence, a relevant choice on $\bar{n}$ should depend on $\varepsilon$, and vanish fast enough as $\varepsilon$ vanishes. Here, we show that we can study the limiting behavior of $n_{\varepsilon}$, and derive the dynamics of the 'fittest trait' in the special case where we scale it as

$$
\bar{n}_{\varepsilon}=e^{\bar{\varphi} / \varepsilon}
$$

for some negative constant $\bar{\varphi}<0$. The motivation for this particular scale will be clear below and, other arguments presented farther indicate that other scales might arise in this problem.

Following the phase function method in asymptotic analysis of reaction-diffusion equations $([1,17,31])$, we use the chain rule in (11), more precisely

$$
\nabla n_{\varepsilon}(t, x)=\frac{n_{\varepsilon}}{\varepsilon} \nabla \varphi_{\varepsilon}, \quad \Delta n_{\varepsilon}(t, x)=\frac{n_{\varepsilon}}{\varepsilon^{2}}\left[\left|\nabla \varphi_{\varepsilon}\right|^{2}+\varepsilon \Delta \varphi_{\varepsilon}\right]
$$

Then equation (6) reads

$$
\frac{\partial \varphi_{\varepsilon}}{\partial t}=R(x)-K_{\sigma} * n_{\varepsilon}-e^{\left(\bar{\varphi}-\varphi_{\varepsilon}\right) /(2 \varepsilon)}+\left|\nabla \varphi_{\varepsilon}\right|^{2}+\varepsilon \Delta \varphi_{\varepsilon}
$$

The interest of the method is that the limit $\varepsilon \rightarrow 0$ does no longer appear to be a singular perturbation as in (6), but a standard limit of a Hamilton-Jacobi equation. We recall that the understanding of the unique viscous limit of Hamilton-Jacobi equations goes back to Crandall and Lions in the 80's (see [12] for a recent survey). It gives the correct notion of weak solution, called viscosity solutions which are unique under very general assumptions.

In the case at hand there are additional difficulties. One of them is due to the unknown coefficients $\alpha_{i}(t)$ arising in the limit. It can be handled as in [16, 2, 3, 7, 23]; there the authors however derive Lipschitz continuity bounds which allow us to extract a subsequence that converges uniformly to obtain a constrained Hamilton-Jacobi equation. The second difficulty come from the singular term $e^{\left(\bar{\varphi}-\varphi_{\varepsilon}\right) /(2 \varepsilon)}$. At points where $\varphi_{\varepsilon}(t, x)>\bar{\varphi}$, this term vanishes and one obtains the usual H.-J. equation. But at points where $\varphi_{\varepsilon}(t, x)<\bar{\varphi}$, then $\frac{\partial \varphi_{\varepsilon}}{\partial t}$ becomes very small (in fact $-\infty$ ) and thus $\varphi_{\varepsilon}$ tends to $-\infty$. Therefore, in the limit we find (as long as there is non extinction otherwise we loose the constraint below)

$$
\left\{\begin{array}{l}
\frac{\partial \varphi}{\partial t}-R(x)+\sum_{i} \alpha_{i} K_{\sigma}\left(x-x_{i}(t)\right)-|\nabla \varphi|^{2} \leq 0 \\
\frac{\partial \varphi}{\partial t}-R(x)+\sum_{i} \alpha_{i} K_{\sigma}\left(x-x_{i}(t)\right)-|\nabla \varphi|^{2}=0, \quad \text { in the set }\{\varphi(t, x)>\bar{\varphi}\} \\
\varphi(t, x)=-\infty \quad \text { in the set }\{\varphi(t, x)<\bar{\varphi}\} . \\
\max _{x} \varphi(t, x)=0, \quad \forall t>0 .
\end{array}\right.
$$

Additionally the concentration points in (10) are exactly the points where $\varphi(t, x)$ vanishes

$$
\varphi\left(t, x_{i}(t)\right)=0
$$

We recall that the difficulty which is still not solved in this case stems from polymorphism, i.e., $\varphi(t)$ can vanish at several points. For the monomorphic situations, the uniqueness is better understood $([2,3])$. The additional difficulty of the above problem, a kind of variational inequality with a H.-J. equation that holds as an equality only in some range of values of the solution itself seems, also to be an interesting theoretical problem.

We have performed numerical experiments illustrating this theoretical limit. The computational domain is $x \in(-1,1)$ (and densities are extended by zero outside this interval) and is discretized on a uniform grid 
with 2000 points (which we have checked are enough for these tests). We have used the invasion exponent given by formula (4) and the gaussian competition kernel in (5) with $\sigma=1 / 8$. Four numerical solutions, are depicted in Figures 5-8 for four values of the survival threshold $\bar{\varphi}$, corresponding to the values of $\bar{n}$ shown in Figure 2 (left the isovalues of the density $n_{\varepsilon}(t, x)$ and right the phase function $\varphi_{\varepsilon}\left(x, t_{\text {final }}\right)$ at the end of the simulation. Notice that the final times are not the same; for $\bar{\varphi}=3.510^{-3}$ in Figure 5 it is $T_{\text {final }}=50$, in Figure 6 for $\bar{\varphi}=4.2510^{-3}$ it is $T_{\text {final }}=40$, in Figure 7 for $\bar{\varphi}=5.2510^{-3}$ it is $T_{\text {final }}=35$ again and in Figure 8 for $\bar{n}=0$ it is $T_{\text {final }}=12$. As expected the higher is the survival threshold, the slower is the trait dynamic, and the higher the selection acts leading to simpler branching patterns (including monomorphism in the first test). In all cases we have truncated the phase function at $4 * \bar{\varphi}$ because we obtain numerical values which are extremely large negative when the theoretical analysis gives $\varphi_{\varepsilon}=-\infty$.

\section{Models with mutation kernels}

More usual models in population biology take into account mutations using integral kernels. They have the advantage to better describe the possible distinction between rare and small mutations and they have been established and used for along time [4]. They have been used in various fields; population systems with adults and juveniles are considered in [5,7], chemostat models can be found in [16], evolution of mutualism in $[18,21]$ and a general analysis of such models in [13]. A mutation probability $M$ is introduced on the space of traits and one may isolate birth terms undergoing mutations. Then, the mutations are modeled by the term $\int b(y) M(x-y) n(t, y) d y$ and (2) leads to

$$
\frac{\partial}{\partial t} n(t, x)=\left(R(x)-K_{\sigma} * n(t, .)\right) n(t, x)-d \sqrt{\bar{n} n(t, x)}+\int b(y) M(x-y) n(t, y) d y .
$$

For simplicity we consider that the trait $x$ is a real number, otherwise the mutation term has to include technical boundary effects as, e.g., in [7]. In this section we show that the analysis we have performed in section 4 can be extended to the present model.

We begin with rescaling the equation (15) in order to introduce again the idea that mutations are small (but not rare, this more elaborated model permits the difference) and, again, a long observation time is needed for the evolution. We arrive as in $[16,2,7]$ to

$$
\varepsilon \frac{\partial}{\partial t} n_{\varepsilon}(t, x)=\left(R(x)-K_{\sigma} * n_{\varepsilon}(t, .)\right) n_{\varepsilon}(t, x)-d \sqrt{\bar{n}_{\varepsilon} n_{\varepsilon}(t, x)}+\int b(y) M_{\varepsilon}(x-y) n_{\varepsilon}(t, y) d y .
$$

with

$$
M_{\varepsilon}(z)=\frac{1}{\varepsilon} M\left(\frac{z}{\varepsilon}\right), \quad M(\cdot) \text { a probability kernel. }
$$

The mathematical analysis can be performed in the same range of scale for $\bar{n}_{\varepsilon}$; we still expect the limit

$$
n_{\varepsilon}(t, x) \underset{\varepsilon \rightarrow 0}{\longrightarrow} n(t, x)=\sum_{i} \alpha_{i}(t) \delta\left(x-x_{i}(t)\right) .
$$

Following the phase function method, we set again

$$
n_{\varepsilon}(t, x)=e^{\varphi_{\varepsilon}(t, x) / \varepsilon}, \quad \bar{n}_{\varepsilon}=e^{\bar{\varphi} / \varepsilon},
$$

Inserting this ansatz in (16), we arrive at

$$
\frac{\partial \varphi_{\varepsilon}}{\partial t}=R(x)-K_{\sigma} * n_{\varepsilon}-e^{\left(\bar{\varphi}-\varphi_{\varepsilon}\right) /(2 \varepsilon)}+\int b(x+\varepsilon z) M(z) e^{\left(\varphi_{\varepsilon}(t, x+\varepsilon z)-\varphi_{\varepsilon}(t, x)\right) / \varepsilon} d z
$$

where we have performed the change of variables $y=x+\varepsilon z \rightarrow z$ in the integral kernel. The new point here, compared to the derivation of (14), is the hamiltonian arising from the integral mutation kernel. Following [16], we write

$$
\int b(x+\varepsilon z) M(z) e^{\left(\varphi_{\varepsilon}(t, x+\varepsilon z)-\varphi_{\varepsilon}(t, x)\right) / \varepsilon} \approx b(x) \int M(z) e^{z \cdot \nabla \varphi_{\varepsilon}(t, x)} d z
$$


Therefore we define the convex hamiltonian

$$
H(p)=\int M(z) e^{z \cdot p} d z
$$

This more complicated hamiltonian is the only modification in the limiting process described in section 4 and thus, we now arrive at

$$
\left\{\begin{array}{l}
\frac{\partial \varphi}{\partial t}-R(x)+\sum_{i} \alpha_{i} K_{\sigma}\left(x-x_{i}(t)\right)-b(x) H(\nabla \varphi(t, x)) \leq 0, \\
\frac{\partial \varphi}{\partial t}-R(x)+\sum_{i} \alpha_{i} K_{\sigma}\left(x-x_{i}(t)\right)-b(x) H(\nabla \varphi(t, x))=0, \quad \text { in the set }\{\varphi(t, x)>\bar{\varphi}\} \\
\varphi(t, x)=-\infty \quad \text { in the set }\{\varphi(t, x)<\bar{\varphi}\} . \\
\max _{x} \varphi(t, x)=0, \quad \forall t>0 .
\end{array}\right.
$$

This constrained variational inequality plays the same role, for integral kernels, than equation (14) in case of mutations described by a diffusion.

\section{Perspectives}

The introduction of survival threshold is rather simple in deterministic population models of adaptive dynamics. An additional mortality rate, under the form of the square root of the population density, has the effect of limiting unrealistic tails corresponding to population below a certain survival density. Numerical results show that, at each time, the population has a finite range of possible traits, and concentrates around fittest traits. Several artifacts of population models disappear and one can observe that jumps do not occur with a survival threshold, that the dynamics of evolution of the trait is much slower and that smooth branching occur that are comparable to the branching patterns arising in direct Monte-Carlo simulations of individual centered stochastic models. Typically, the number of branching points increases as the survival threshold diminishes, a phenomena that has been already explained in terms of the demographic stochasticity ([11]), the notion in stochastic models corresponding to our survival threshold.

In terms of asymptotic analysis, this has lead us to introduce such small parameters: the mutation rate $\varepsilon$ and this survival threshold $\bar{n}_{\varepsilon}$. In Section 4 we have introduced an exponential scale for $\bar{n}_{\varepsilon}$, see formula (12). This distinguished limit allows us to derive a rigorous asymptotic analysis compatible both with concentrations of the population and the zero densities replacing usual tails in deterministic population models. This asymptotic analysis is compatible with the formalism introduced in [16] and leads again to a Constrained Hamilton-Jacobi equation and thenew feature is that it takes the form of a variational inequality.

In view of its importance as already underlined in [11], a natural question concerns the transition from monomorphism to dimorphism, from dimorphism to trimorphism...etc Our formalism allows us to compute numerically the relations between the two small parameters when these transitions occur. We have performed this computation and we present it under the form of two corresponding transition curves $\varepsilon \mapsto \bar{n}_{\varepsilon}$ for the transitions of a single to two branches and from two to three branches. In log-log scale they are surprisingly parallel lines with slopes which we estimate to be 1.45. The results are depicted in Figure 9. This seems to indicate that other possible distinguished limits in (6), different from that we have analyzed theoretically in this paper, may be possible, leading certainly to a completely different analysis.

A natural follow-up of our study would also be to treat specific problems and to calibrate the mutation rate and survival threshold on empirical data.

\section{References}

[1] Barles G., Evans L. C. and Souganidis P. E., Wavefront propagation for reaction diffusion systems of PDE, Duke Math. J. 61 (1990) 835-858.

[2] Barles, G. and Perthame, B., Concentrations and constrained Hamilton-Jacobi equations arising in adaptive dynamics. In Recent Developments in Nonlinear Partial Differential Equations, D. Danielli editor. Contemp. Math. 439 (2007), 57-68. 
[3] Barles, G. and Perthame, B., Dirac concentrations in Lotka-Volterra parabolic PDEs. Indiana Univ. Math. J. To appear.

[4] Bürger, R. The mathematical theory of selection, recombination and mutation. Wiley (2000).

[5] Calsina, À, Cuadrado, S., Small mutation rate and evolutionarily stable strategies in infinite dimensional adaptive dynamics. J. Math. Biol. 48 (2004) 135-159.

[6] Calsina, À, Perelló, C. Equations for biological evolution. Proc. Roy. Soc. Edinburgh Sect. A 125 (1995), $939-958$.

[7] Carrillo, J. A., Cuadrado, S. and Perthame, B., Adaptive dynamics via Hamilton-Jacobi approach and entropy methods for a juvenile-adult model. Mathematical Biosciences, 205(1) (2007), 137-161.

[8] Champagnat, N., A microscopic interpretation for adaptive dynamics trait substitution sequence models. Stoch. Processes and their Appl. 116 (2006) 1127-1160.

[9] Champagnat, N., Ferrière, R. and Ben Arous, G. The canonical equation of adaptive dynamics: a mathematical view. Selection 1-2, 73-83 (2001).

[10] Champagnat, N., Ferrière, R. and Méleard, S., Unifying evolutionary dynamics: from individual stochastic processes to macroscopic models. Theoretical Population Biology, 69 (2006), No. 3, 297-321.

[11] Claessen, D., Andersson, J., Persson, L. and de Roos, A. M., Delayed evolutionary branching in small populations. Evolutionary Ecology Research 9 (2007) 51-69.

[12] Crandall, M. G., Ishii, H. and Lions, P.-L., User's guide to viscosity solutions of second order partial differential equations, Bull. Amer. Math. Soc. 27 (1992), 1-67.

[13] Desvillettes, L., Jabin, P.-E., Mischler, S. and Raoul, G., On mutation selection dynamics. Preprint (2007).

[14] Dieckmann U. and Law R., The dynamical theory of coevolution: A derivation from a stochastic ecological processes. J. Math. Biol. 34 (1996) 579-612.

[15] Diekmann, O., Beginner's guide to adaptive dynamics. Banach Center Publications 63 (2004) 47-86.

[16] Diekmann, O., Jabin, P.-E., Mischler, S. and Perthame, B., The dynamics of adaptation : an illuminating example and a Hamilton-Jacobi approach, Th. Pop. Biol., 67(4) (2005) 257-271.

[17] Evans, L.C. and Souganidis, P.E., A PDE approach to geometric optics for certain reaction-diffusion equations. Indiana Univ. Math J. 38 (1989), 141-172.

[18] Ferrière R., Bronstein J.-L., Rinaldi, S., Law, R. and Gauduchon, M., Cheating and the evolutionary stability of mutualism, Proceedings of the Royal Society of London Series B 269 (2002) 773-780.

[19] Fleming W. H., Soner H. M., Controlled Markov processes and viscosity solutions. Applications of Mathematics 25, Springer (1993)

[20] Fournier, N. and Méleard, S., A microscopic probabilistic description of a locally regulated population and macroscopic approximations. Ann. Appl. Probab. 14 (2004), 1880-1919.

[21] Gauduchon, M., Dynamique adaptative et évolution des mutualismes, PhD Thesis, University Pierre et Marie Curie (2006).

[22] Génieys, S., Volpert, V. and Auger, P., Adaptive dynamics: modeling Darwin's divergence principle. C. R. Acad. Sc. Paris, biologies, 329(11) (2006), 876-881.

[23] Génieys, S. and Perthame, B., Dynamics of Nonlocal Fisher concentration points: a nonlinear analysis of Turing patterns. Mathematical Modeling in Natural Phenomenon, Vol. 2(4) 2007, 135-151.

[24] Geritz S. A. H., Kisdi E., Meszena G. and Metz J. A. J., Evolutionary singular strategies and the adaptive growth and branching of the evolutionary tree. Evolutionary Ecology 12 (1998) 35-57.

[25] Geritz S. A. H., Metz J. A. J., Kisdi E., Meszena G., Dynamics of adaptation and evolutionary branching. Physical Review Letters 78 (1997) 2024-2027.

[26] Legendre. S. ZEN Eco-evolutionary Software, http://www.biologie.ens.fr/ legendre/zen/zen.html

[27] Magal, P., Webb, G. F. Mutation, selection and recombination in a model of phenotype evolution. Discrete Contin. Dynam. Systems 6 No 1 (2000) 221-236.

[28] Metz J. A. J., Geritz S. A. H., Meszna G., Jacobs F. J. A. and van Heerwaarden, J. S., Adaptive dynamics, a geometrical study of the consequences of nearly faithful reproduction. Stochastic and spatial structures of dynamical systems (Amsterdam, 1995), 183-231, Konink. Nederl. Akad. Wetensch. Verh. Afd. Natuurk. Eerste Reeks, 45, North-Holland, Amsterdam, 1996.

[29] Meszéna, G. and Gyllenberg, M., Link between population dynamics and dynamics of darwinian evolution. Phys. Rev. Letters 95, 078105 (4 pages), (2005).

[30] Perthame, B. Transport equations in biology. Series 'Frontiers in Mathematics', Birkhauser (2007).

[31] Perthame B. and Souganidis P. E., Front propagation for a jump process model arising in spatial ecology, DCDS, 13(5), (2005) 1235-1248.

[32] Proulx, S. R. and Day, T. What can invasion analyses tell us about evolution under stochasticity in finite populations? Selection, 1-2, 1-15 (2001).

[33] Wilson, W. G. Resolving discrepencies between deterministic population models and individual-based simulations. American Naturalist 151 No 2 (1998) 116-134. 


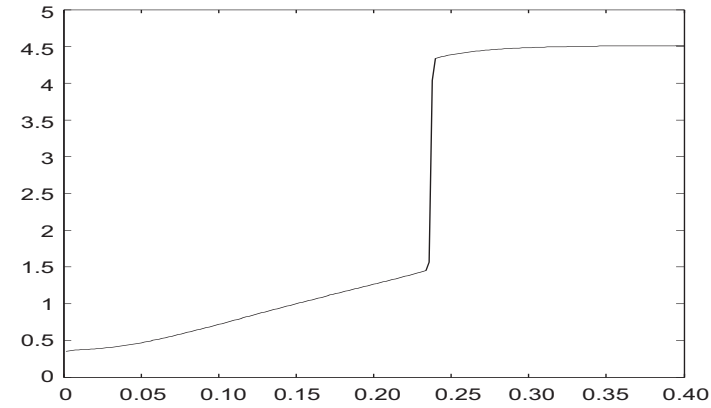

(a)

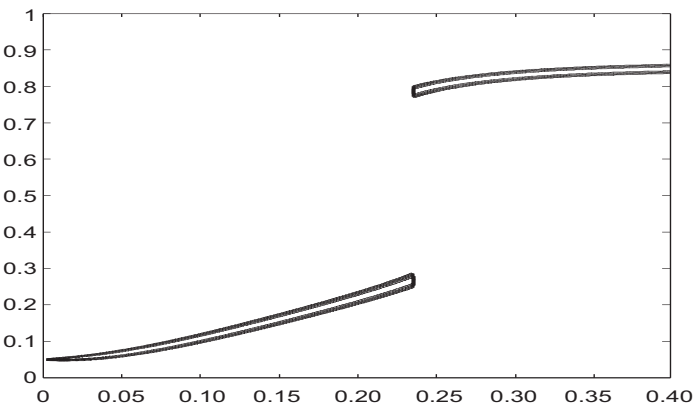

(b)

Figure 1: Typical jump of the fittest trait arising from the sudden growth of a population tail in a standard deterministic model as (1). The abscissae is time. (a) The total population density. (b) The fittest trait (in fact isovalues of the density $n_{\varepsilon}(t, x)$ ). This graphics is borrowed from [2]. 


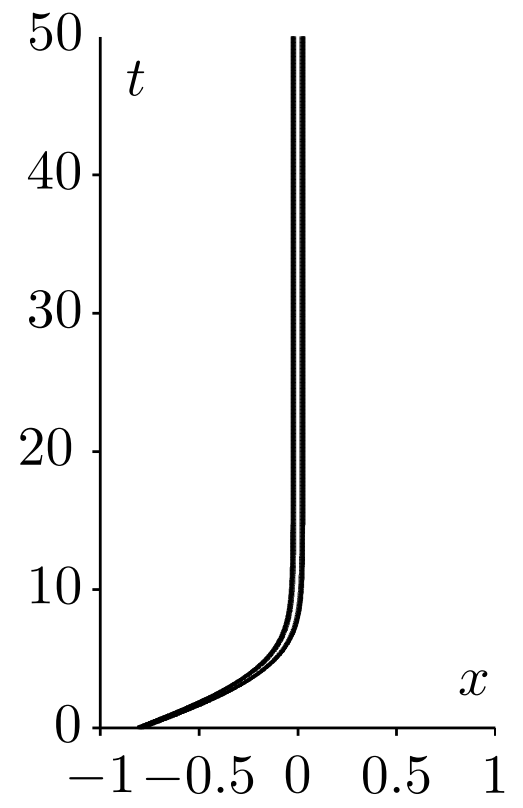

(a)

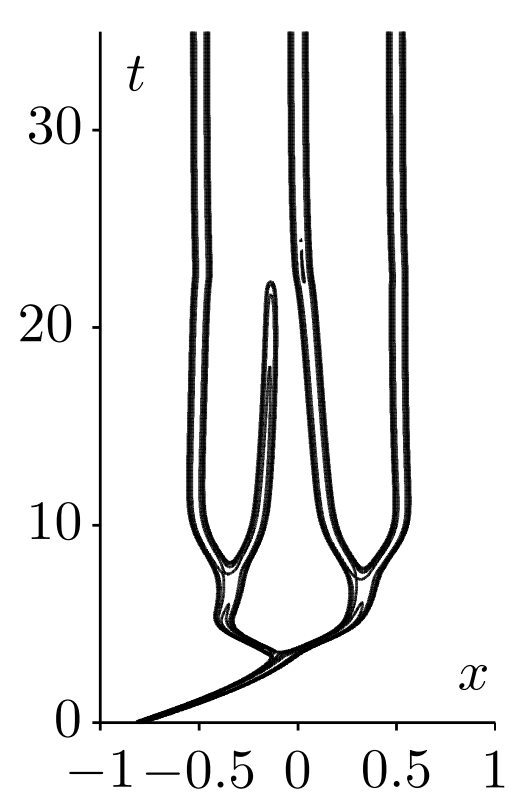

(c)

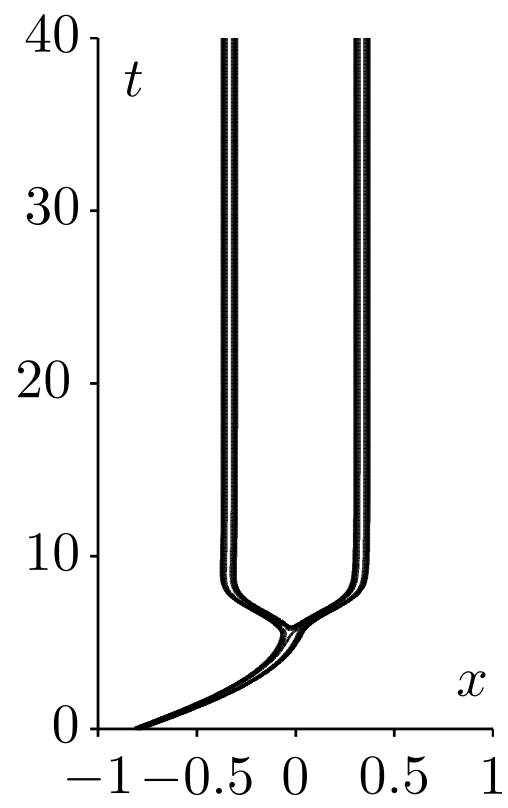

(b)

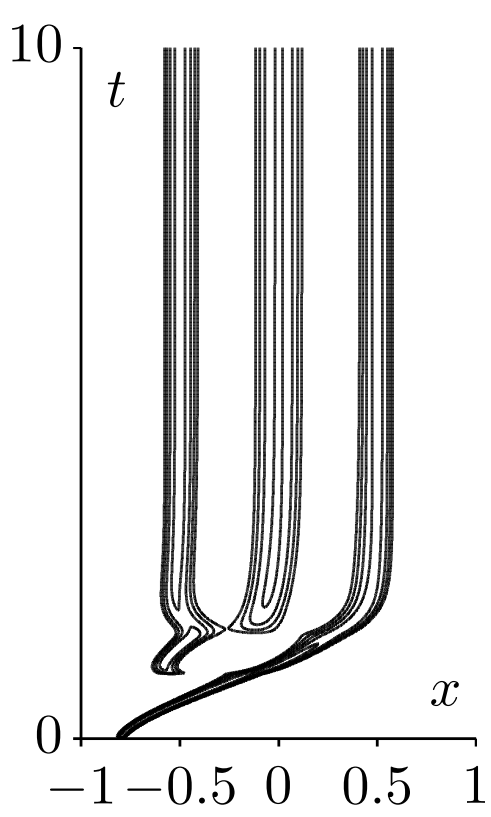

(d)

Figure 2: Isovalues of the numerical solution to (6) with $\varepsilon=510^{-4}$ and gaussian competition kernel, $\sigma=1 / 8$. The abscissae is the trait $x$ and ordinate axis is time. Four different values of $\bar{n}$ are used, larger from (a) to (d): (a) $\bar{n}=9.110^{-4}$, (b) $\bar{n}=2.010^{-4}$, (c) $\bar{n}=2.810^{-5}$, (d) $\bar{n}=0$. Evolution speed as well as the branching pattern are strongly affected by $\bar{n}$ value. Final times are respectively, (a) $t_{\text {final }}=50$, (b) $t_{\text {final }}=40$, (c) $t_{\text {final }}=35$, (d) $t_{\text {final }}=10$. 


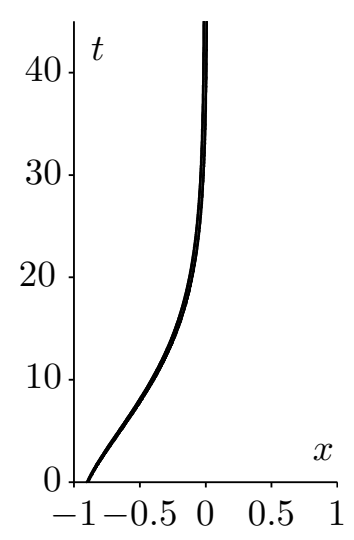

(a)

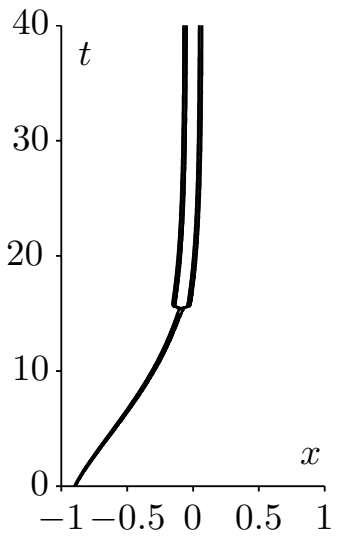

(b)

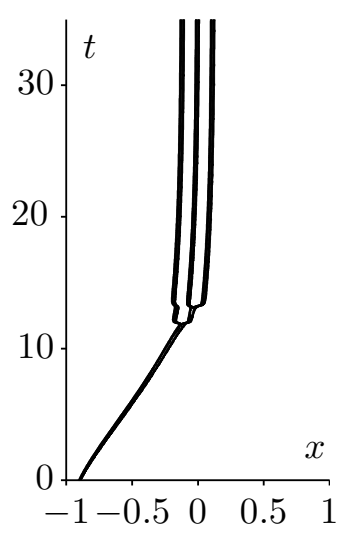

(c)

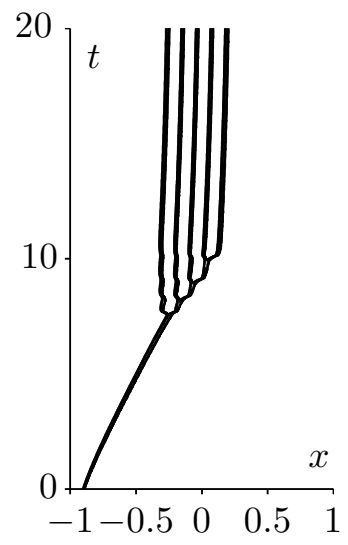

(d)

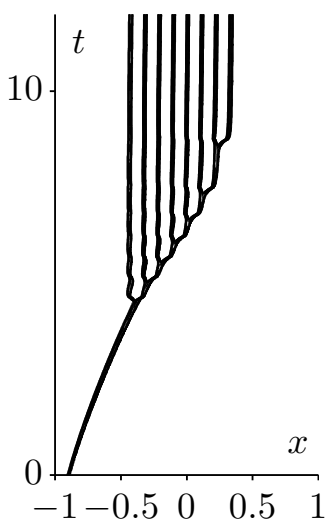

(e)

Figure 3: Isovalues of the numerical solution to (6) with $\varepsilon=510^{-4}$ and quadratic competition kernel, $\sigma=1 / 10$. Five different values of $\bar{n}$ are used, smaller from (a) to (e). The abscissae is the trait $x$ and ordinate axis is time. Final times are respectively, (a) $t_{\text {final }}=45$, (b) $t_{\text {final }}=40$, (c) $t_{\text {final }}=35$, (d) $t_{\text {final }}=20$, (e) $t_{\text {final }}=12$. 


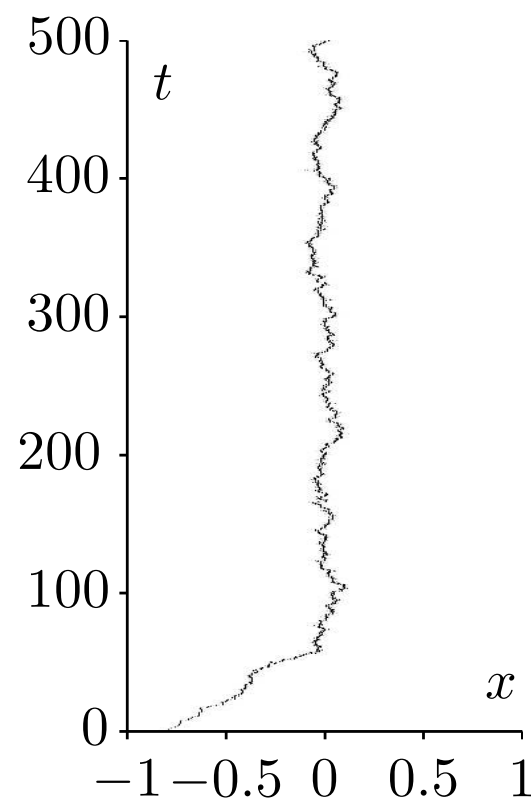

(a)

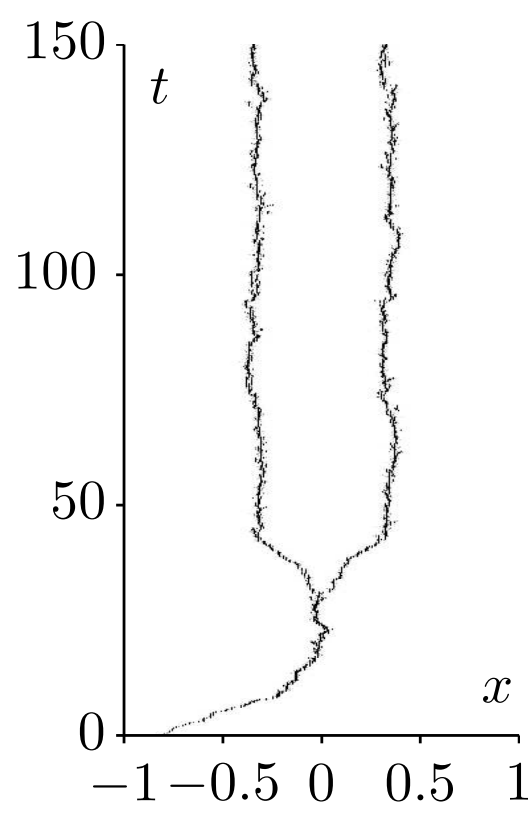

(b)

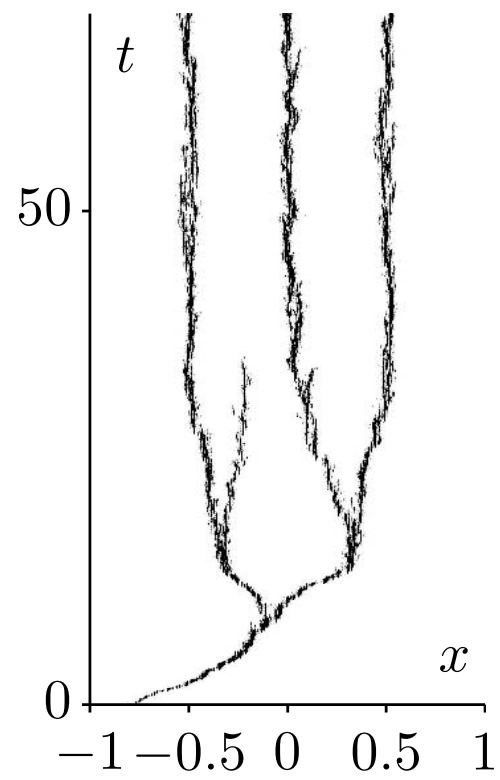

(c)

Figure 4: Stochastic simulations of individual based model of evolutionary dynamics compatible with (6) in particular gaussian competition is used. Three different values of a parameter related to the population size are chosen ; (a) $\mathrm{K}=200$, (b) $\mathrm{K}=1000$, (c) $\mathrm{K}=5000$. Compare with Figure 2. 


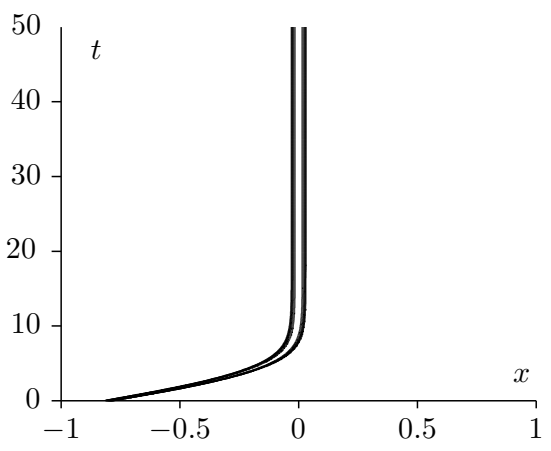

(a)

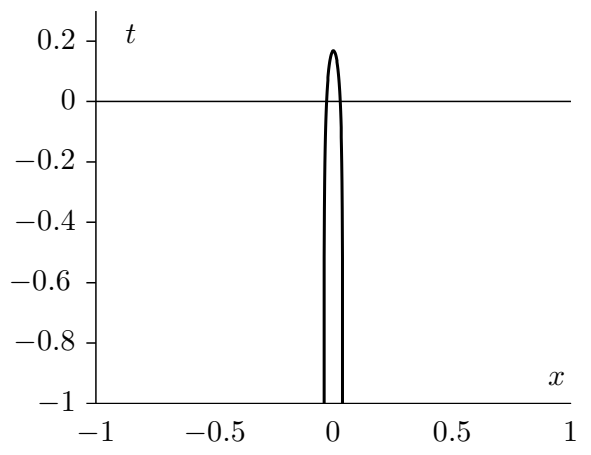

(b)

Figure 5: Numerical solution to (6) with $\varepsilon=510^{-4}, \sigma=1 / 8$ and $\bar{\varphi}=-3.510^{-3}$. (a) the isovalues of $n_{\varepsilon}$, the $y$ axis is time. (b) the 'phase' function $\varphi_{\varepsilon}$ in (11) at the final time $t_{\text {final }}=50$, the unit on the y-axis is $10^{-2}$.

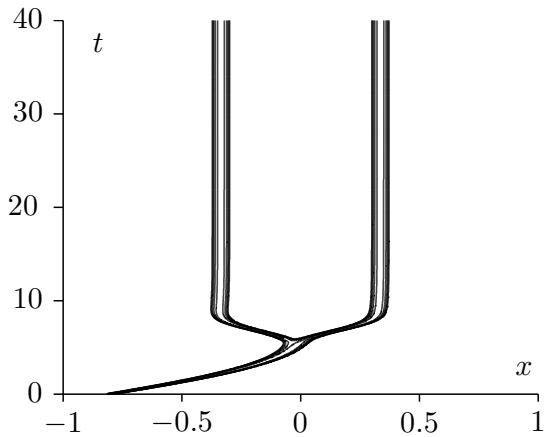

(a)

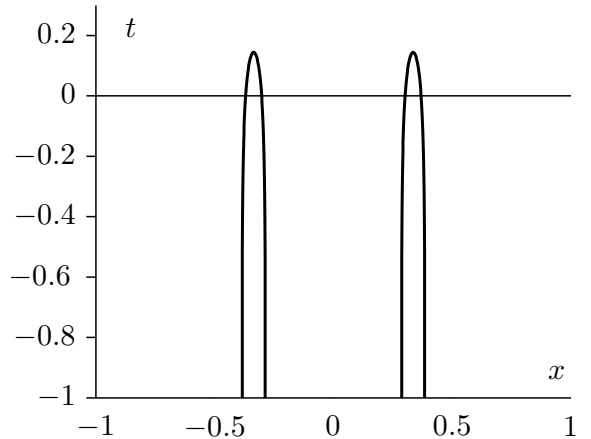

(b)

Figure 6: Same as Figure 5 with $\bar{\varphi}=-4.2510^{-3}$. For the 'phase' function $\varphi_{\varepsilon}$ in (11) the final time $t_{f i n a l}=40$ and the unit on the $\mathrm{y}$-axis is $10^{-2}$. 


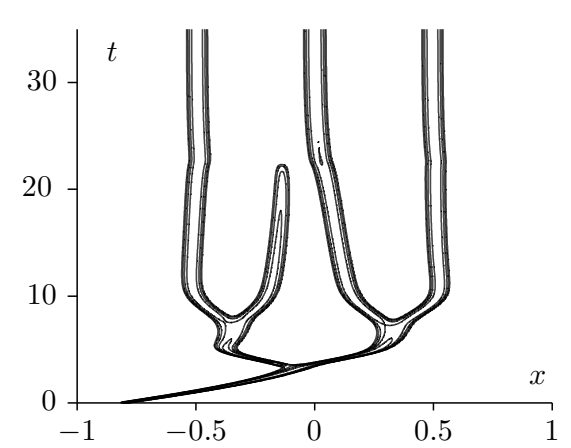

(a)

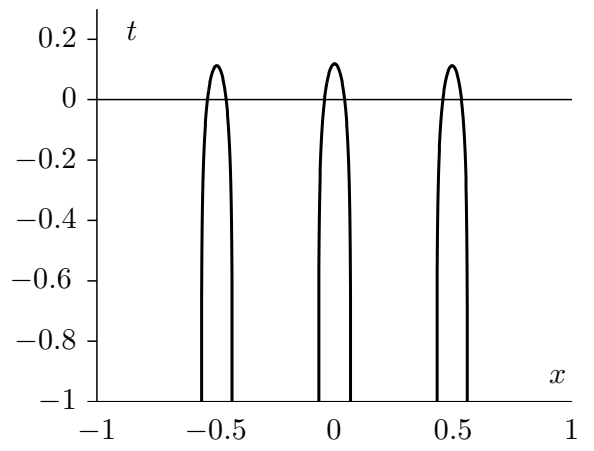

(b)

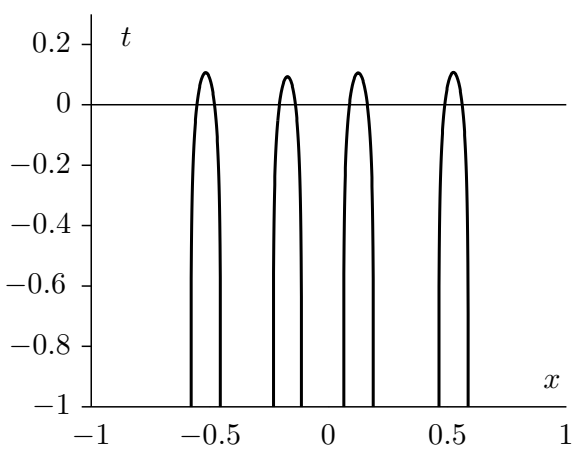

(c)

Figure 7: Same as Figure 5 with $\bar{\varphi}=-5.2510^{-3}$. (b) the 'phase' function $\varphi_{\varepsilon}$ in (11) at the final time $t_{\text {final }}=35$. (c) the 'phase' function $\varphi_{\varepsilon}$ in (11) at the intermediate time $t=12$, the unit on the y-axis is $10^{-2}$.

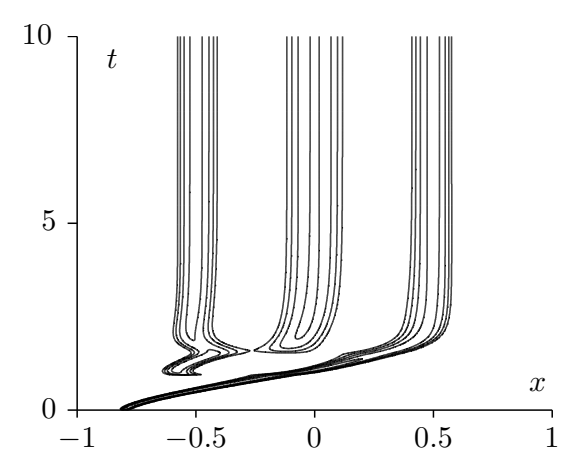

(a)

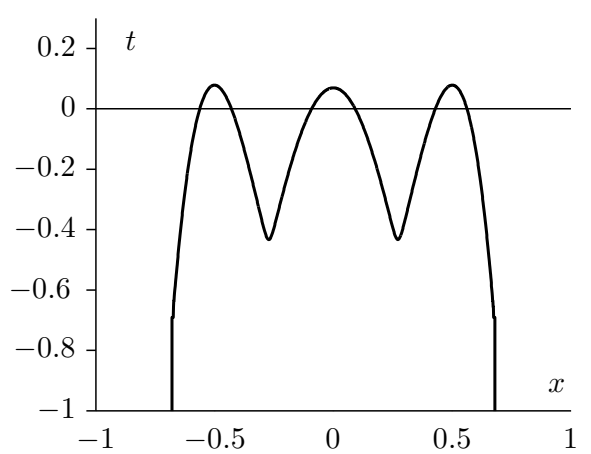

(b)

Figure 8: Same as Figure 5 with $\bar{\varphi}=-\infty$. Again notice that, for the 'phase' function $\varphi_{\varepsilon}$ in $(11)$ the final time is $t_{f i n a l}=12$ and the unit on the y-axis is $10^{-2}$. 


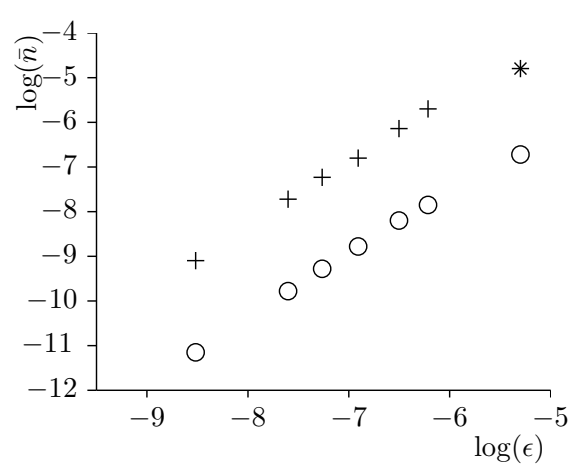

(a)

Figure 9: Other possible distinguished scales in the Lotka-Volterra model (3) with $\sigma=1 / 8$. The points indicate how the survival threshold $\bar{n}_{\varepsilon}$ depends on the mutation rate $\varepsilon$ in a $\log -\log$ scale. The first line $(+)$ represents the transition form monomorphism to dimorphism. The second line (o) represents the transition from two to three branches. The $\left(^{*}\right)$ point on the first line represents a direct transition from extinction to dimorphism 